\title{
A Proposed Genome of Mobile and Situated Crowdsourcing and Its Design Implications for Encouraging Contributions
}

\author{
Yun Huang ${ }^{\mathrm{a}}$, Alain Shema ${ }^{\mathrm{a}}$, Huichuan Xia ${ }^{\mathrm{a}}$ \\ ${ }^{a}$ School of Information Studies, Syracuse University
}

\begin{abstract}
A number of papers have surveyed mobile crowdsourcing systems and, to a lesser extent, situated crowdsourcing systems. These surveys have either contributed a comprehensive taxonomy of the diverse application domains where the systems have tapped into or characterized different components of the system platforms. In this paper, we present a survey of mobile and situated crowdsourcing systems by addressing fundamental questions about user contributions that system designers pose when building new systems or evaluating existing ones. We select and analyze 40 mobile and situated crowdsourcing systems that had prototypes deployed and studied in the real world. Inspired by the MIT's genetic model of collective intelligence, we propose our genetic model and new genes for mobile and situated crowdsourcing systems by examining user contributions to the selected systems. We present the observed patterns of different genes and discuss how the model can be used to create new applications and design ideas. We also provide examples to illustrate how the unique genes inspire new design suggestions for encouraging user contributions in the context of mobile and situated crowdsourcing by reviewing the existing design principles of online communities.

Keywords: Survey, Mobile Crowdsourcing, Situated Crowdsourcing, Genome, Design Suggestions
\end{abstract}

Email addresses: yhuang@syr.edu (Yun Huang), sralain@syr.edu (Alain Shema), hxiaesyr.edu (Huichuan Xia)

Preprint submitted to Elsevier

July 16, 2016

(C) 2016. This manuscript version is made available under the Elsevier user license http://www.elsevier.com/open-access/userlicense/1.0/ 


\section{Introduction}

Mobile devices and wearable technologies equip people with unprecedented computing and sensing capabilities, allowing people to form powerful networks that enable pervasive information gathering and dissemination. Mobile crowdsourcing has been leveraging these platforms and emerged as an effective way of harnessing the collective intelligence of mobile crowds [1, 2, 3, 4]. Meanwhile, situated crowdsourcing is emerging as a new form of crowdsourcing that also taps into the power of mobile crowds $[5,6,7]$. Unlike mobile crowdsourcing where people usually need to install an application on their own smart devices or carry a mobile sensor with them, situated crowdsourcing invites people to use embedded input mechanisms in public places, e.g., through public displays, tablets, etc. This can lower the barrier for potential contributors to make contributions with less privacy concerns (e.g., location tracking, phone usage logging, etc.) and without consuming their data plans and phone battery [8]. Situated crowdsourcing systems also offer a better way of targeting potential contributors by setting up the displays where the desired contributors are most likely to be found (e.g., school for students, malls for shoppers, etc.).

Given the proliferation of mobile and situated crowdsourcing systems, people seek both systematic approaches and design principles to address the design questions behind these systems. The MITs genetic model [9] provides us an effective tool to systematically approach the design of systems that rely on collective intelligence. In particular, the model starts by answering four major questions: what is being done, who is doing it, why are they doing it, and how is it being done. After studying a large number of web-based collective intelligence systems, sixteen principle genes were discovered. When people design a new collective intelligence system, they can look at the four questions and identify the genes associated with their own systems. If their system shares common genes with the existing collective intelligence systems that are successful, they may consider following the footprints of the successful systems by applying their good practices.

In addition, a suite of design principles for encouraging contributions to online communities are nicely presented in Kraut and Resnicks book [10]. More specifi- 
cally, a total of 35 design claims are summarized with different focuses, e.g. providing clarity and targeted requests for contributions and easy-to-use monitoring tools; emphasizing that requests should be specific, simple and with deadlines; identifying appropriate;intrinsic and extrinsic incentive mechanisms; forming smaller and committed online groups, etc.

Mobile and situated crowdsourcing share many common features with collective intelligence and online communities. In principle, their success heavily relies on the availability of the crowds and user generated content (contributions). Therefore, both the genetic model [9] and the design principles of encouraging contributions to online communities [10] provide us rich materials to understand and address the design of mobile and situated crowdsourcing systems.

However, motivating the mobile crowds to make contributions poses new opportunities as well as unique challenges. For example, since users of mobile phones are able to receive notifications and quickly respond to them, opportunities to create new interactions emerge. Some challenges accrue from users short attention span and constant movement, which potentially makes it difficult for users to make contributions of high quality compared to using desktops. Unique challenges also arise when the values of location-based contributions may be invalidated soon after the contributions are made. For example, Tiramisu is a mobile application that crowdsources transit information [11]. If a user reports a bus passing by, the information can only be used to predict real-time bus arrival information for the downstream stops before the bus trip is over.

In this paper, we make the following major contributions. First, inspired by the MIT's genetic model of collective intelligence, we propose six fundamental questions about user contributions that system designers pose when building a new mobile and situated crowdsourcing system or could use to review their current systems. We provide an extensive survey of 40 mobile and situated crowdsourcing systems that had prototypes deployed and studied in the real world through a whole new lens, i.e., by addressing the proposed six questions. By analyzing the survey results, we create a new genetic model and new genes that can be used to characterize mobile and situated crowdsourcing systems. Second, we present interesting observations of the genes and discuss how our model can be used to create new applications and design ideas. We also 
provide examples to illustrate how the unique genes inspire new design suggestions for encouraging user contributions in the context of mobile and situated crowdsourcing by reviewing the existing design principles of online communities.

\section{Related Work}

Our study is built upon reviewing a large number of mobile and situated crowdsourcing systems, as well as examining existing survey results of such systems. In this section, we first review how other surveys present, categorize or analyze these systems. We then provide an overview of different mechanisms that were studied to improve user contributions.

\subsection{Prior Surveys of Mobile and Situated Crowdsourcing}

Extensive surveys have been developed on mobile crowdsourcing systems [1, 2, 3] and crowdsourcing systems in general [4]. Different terms, e.g., participatory sensing or mobile crowdsensing, have been used to refer to mobile crowdsourcing. However, we view them all within the scope of mobile crowdsourcing, where people use mobile phone applications or sensing devices to either explicitly make contributions or implicitly generate content for the systems. The prior surveys reviewed the systems mainly from three perspectives.

One perspective is to categorize systems according to their application domains. For example, seven application areas were identified to which smartphone sensors could contribute: health monitoring, traffic monitoring, commerce, human behavior tracking, application with special purposes, social interaction, and environment monitoring [12]. A similar taxonomy was proposed and includes: environmental monitoring, transportation and traffic planning, urban dynamics sensing, location services, mobile social recommendation, healthcare, and public safety [3]. Under each category, they further summarized systems with subcategories. For instance, public safety has two sub-categories: crime prevention and disaster relief.

Depending on whether users explicitly contributed data or data was collected by sensors automatically, Lane et al. proposed two types of mobile crowdsourcing: par- 
ticipatory sensing and opportunistic sensing [12]. Regarding participatory sensing systems, three models have been proposed: collective design and investigation, public contribution, and personal use and reflection [2]. Similarly, Tilak surveyed 17 participatory sensing applications and classified them into four categories: health and fitness, environmental monitoring, transportation and civil infrastructure monitoring, and urban sensing [13]. With a focus on transportation, Lee and Gerla classified urban vehicular sensing systems into different types, including: street-level traffic flow estimation; proactive urban surveillance; vehicular safety warning services; ride quality monitoring; and location aware microblogging [14].

The second perspective is to analyze the major functional components or technical aspects of these platforms. For example, Fuchs-Kittowski and Faust discussed the architecture of mobile crowdsourcing systems [15]. In their view, a typical architecture includes a campaign organizer who initiates and monitors data collection, participants who make contributions, and end users who access and process the contributions from participants. Furthermore, the architecture is divided into two independent run-time systems: a backend system as the server, and a mobile device as the client portal.

Another perspective is to look at the characteristics of these platforms. For example, Chatzimilioudis et al. proposed a taxonomy of mobile crowdsourcing applications based on whether their platform is web-extend or not, the type of involvement (participatory vs. opportunistic), data wisdom (individual vs. collective), quality of contribution (heterogeneous vs. homogenous), incentive mechanisms (monetary and others), human skills, different types of sensors, and whether they are location sensitive [1].

Similarly, Vukovic et al. presented an overview of crowdsourcing systems by grouping them according to the complexity of the tasks (crowd as sensors vs. crowd as solvers) [16]. They also reviewed the proposed taxonomy from prior work as well as the challenges that they identified in order to harness crowds. These challenges include incentive mechanisms for crowds to contribute, and quality control and verification schemes of contributions. However, these are not done in a systematic manner, but the work is rather a review of prior work that deals with ubiquitous crowdsourcing systems.

Our work draws from the existing literature and proposes a new model that can 
address the various perspectives by answering a set of fundamental questions that are critical to system design. Similar to the purpose of the MIT's genetic model of collective intelligence [9], our mobile and situated crowdsourcing genome can be provided to system designers to examine their systems by borrowing ideas from the design of those successful systems that have the same genes as theirs.

\subsection{Design Approaches to Encouraging User Contributions}

As mentioned above, the design principles proposed for encouraging user contributions to online communities provide rich materials for mobile and situated crowdsourcing [10]. However, they need to be tested in mobile and situated crowdsourcing environments.

Several studies on how to encourage user contribution to mobile crowdsourcing systems could resonate with a few design claims in [10], though they were not developed in a systematic way.

For example, Tiramisu is a mobile crowdsourcing transit information system where riders share information about their bus rides. This information is used to generate real-time bus arrival schedules. A 10-month field experiment showed that a quid-proquo approach, blocking people from reviewing real-time bus schedules for not making sufficient contributions, effectively increased users' contributions [17]. However, presenting the request explicitly to users seemed not effective in encouraging contributions. This was not quite consistent with the first design claim presented in [10], where "making the list of needed contributions easily visible increases the likelihood that the community will provide them". Possibly, this is because bus-arrival information is time sensitive, thus the quid-pro-quo approach is more effective in impacting people's behaviors than simply sending a contribution request.

Another work found that reducing contribution time and effort and making it as easy and as natural as possible could motivate people to make more contributions [18]. They designed a mechanism that when users swiped and unlocked their phones, they could complete a mini crowdsourcing task, e.g., rating a cloud picture. Such a design echoed the second design claim presented in [10] that "Easy to use tools for finding and tracking work that needs to be done will increase the amount that gets done". 
Money (or a virtual currency) has been found to be a strong incentive in both online community and crowdsourcing systems. For example, a mobile crowdsourcing system was created to find parking services and it embeds a game-theoretic incentive mechanism [19]. Users who contribute parking information would be rewarded with certain amount of system credits. This was especially true for systems that were at the early stage of crowdsourcing [20]. Money could be an essential incentive for people to participate and contribute, and paid crowdsourcing might generate better results and completion time than non-paid. These findings in mobile crowdsourcing systems partially resonate with the $23 \mathrm{rd}$ design claim presented in [10], i.e., "Rewards, whether in the form of status, privileges, or material benefits, will motivate contributions."

In brief, although a few prior works studied different designs to encourage user contributions, a systematic approach to understand the design issues in the context of mobile and situated crowdsourcing is still lacking. Our work aims to address this gap by providing a model as a tool to help address design issues.

\section{The Genome of Mobile and Situated Crowdsourcing}

Inspired by the MIT genome for collective intelligence [9], we proposed six fundamental questions about user contributions and created a new genetic model for mobile and situated crowdsourcing by examining these questions using 40 real systems. In this section, we first present our methods for creating the model in more details, and then introduce the new model and its genes.

\subsection{Method}

Creating the new genetic model for mobile and situated crowdsourcing involved the following major steps: selecting systems, defining questions, and creating new genes by examining the questions with the selected systems.

To select representative systems, we started by examining survey papers on mobile and situated crowdsourcing systems, including [1], [3], [12], [13], and [15]. We reviewed the systems discussed in those papers and selected the ones that were deployed 
in real world. We also added recent systems that were not included in the prior surveys. Finally, we identified 40 mobile and situated crowdsourcing systems that had prototypes or were tested in the real world.

To define the questions, we looked at the four questions (of what, who, why and how) proposed in the MIT genome for collective intelligence [9]. The MIT model was geared toward managers and was meant to help them decide whether a task should be crowdsourced, and the questions were defined to help identify different mechanisms (e.g., collection, contest, or collaboration) for accomplishing a task through collective intelligence. As we meant to understand user contributions, we defined four new questions centered on user contributions, including: what is the contribution about, who is contributing and/or benefiting from the contributions, why are they contributing, and how are the contributions generated. Since user's mobility, time and location factors could challenge their contributions to mobile and situated systems [21, 22, 23], we also created two new questions, where is the contribution valid, and when is the contribution is valid? These two questions are critical and helpful when evaluating the constraints faced by users when contributing to mobile and situated crowdsourcing systems.

To create the genes, all three researchers first reviewed 20 systems by discussing how to answer the six questions together. If some classifications provided in the prior surveys could be applied to create the genes, then we leveraged the existing classifications, so that our work can be easily integrated with prior findings. If the existing classifications were not sufficient to answer a question, we proposed a new classification (i.e. gene). For instance, in one survey, mobile crowdsourcing applications are classified into two types: environment-centric and people-centric [15], which could be used to answer the question of what the contribution is about. When examining the Laermometer [24] system, which used microphones and GPS on smartphones to collect information about noise levels, we could assign the environment-centric gene, indicating the contribution is about the environment. However, txteagle is about translating and/or transcribing documents [25]. It requires users to perform some computation (e.g., translating a document) before making a contribution. We did not find any classification from the existing surveys that best described this type of contribution. Thus, 
we created service-centric as a new gene.

Similarly, intrinsic and extrinsic motivations were heavily discussed in prior literature, including [10]. Given that different application domains discussed the two motivations quite differently, we posited that intrinsic and extrinsic would be the best descriptions to fit for the variations of the why gene. In terms of how the contributions are generated, prior survey work on mobile crowdsourcing systems [1] also identified two approaches, i.e., participatory and opportunistic. We used these two terms to designate the genes that constitute the how question.

In brief, we created a total of 14 genes for mobile and situated crowdsourcing by leveraging 6 classifications from existing survey work $[1,3,12,13,15]$ and defining 8 new classifications.

After creating the genes by examining all the questions with the 20 systems, two researchers independently answered these questions for the remaining 20 systems. The third researcher reviewed the answers and addressed the disagreement with the other two researchers together. Overall, we reached a high level of agreement on assigning the genes to the systems, e.g., with $80 \%$ for why and when genes, $85 \%$ for who and how, $90 \%$ for where, and $95 \%$ for what genes.

\subsection{Definitions of the Genes}

Table 1 summarizes the new genes of mobile and situated crowdsourcing and their brief definitions. We provide detailed descriptions of the genes as follows.

\subsubsection{The What Genes}

In terms of what the contribution is about, we identified three major answers:

- environment-centric: Usually involves sensing, monitoring and reporting information about the surrounding environment, including noise, traffic, road condition, parking space, etc. For instance, ParkNet [26] is a system equipped in a user's car and can automatically detect whether there is available parking space.

- people-centric: Usually refers to the collection of information about the person and the collection of contributions are through smart devices, e.g. phones, 


\begin{tabular}{|c|c|c|}
\hline Question & Gene & Useful When ... \\
\hline \multirow{3}{*}{ What } & Environment-centric (10) & $\begin{array}{l}\text { Contribution is an observation of the environment, such } \\
\text { as traffic, noise, road condition or safety condition. }\end{array}$ \\
\hline & People-centric (9) & $\begin{array}{l}\text { Contribution is an observation of people themselves, e.g., } \\
\text { health, life, behaviors, or society. }\end{array}$ \\
\hline & Service-centric (21) & $\begin{array}{l}\text { Contribution is a service that may need either contribu- } \\
\text { tion or labor, e.g., document translation, driving, etc. }\end{array}$ \\
\hline \multirow{3}{*}{ Who } & Crowd (14) & There is no explicit requester. \\
\hline & Requester/Contributor (21) & A contribution directly responds to an explicit request. \\
\hline & Practitioner/Crowd (5) & $\begin{array}{l}\text { Contributions are used by practitioners (researchers) to } \\
\text { make practical changes (advance knowledge). }\end{array}$ \\
\hline \multirow[t]{2}{*}{ Why } & Intrinsic (18) & $\begin{array}{l}\text { Contributors are motivated by making the contribu- } \\
\text { tions themselves independent of other downstream con- } \\
\text { sequences. }\end{array}$ \\
\hline & Extrinsic (22) & $\begin{array}{l}\text { Contributors are motivated by external rewards, e.g. } \\
\text { money, valuable objects, reciprocity, etc. }\end{array}$ \\
\hline \multirow{2}{*}{ How } & Participatory (32) & Contributions are actively created by the contributors. \\
\hline & Opportunistic (11) & $\begin{array}{l}\text { Contributions are generated and computed from various } \\
\text { sensors of mobile devices. }\end{array}$ \\
\hline \multirow[t]{2}{*}{ Where } & Local (26) & $\begin{array}{l}\text { Requires or favors users to make contributions from a cer- } \\
\text { tain area or a particular location. }\end{array}$ \\
\hline & Anywhere (14) & Contributions can be made at any place. \\
\hline \multirow[t]{2}{*}{ When } & Time-bound (16) & $\begin{array}{l}\text { Requires users to make contributions within a certain pe- } \\
\text { riod of time and/or the contribution has a limited lifespan. }\end{array}$ \\
\hline & Anytime (24) & $\begin{array}{l}\text { Contributions can be made anytime and have a longer } \\
\text { lifespan. }\end{array}$ \\
\hline
\end{tabular}

Table 1: The Genome Table. The number in the parentheses shows the number of systems having the corresponding gene. Three systems have both of the How genes. 
watches or other sensors. This information can be about their behavioral patterns (driving habits, etc.), their health indicators (heart rate), or physical exercising habits (number of steps, exercise intensity, etc.). For instance, Social Drive [27] gathers users' driving behavioral data and recommends for them the optimal driving habits for fuel economy in specific trips.

- service-centric: Usually involves an explicit request for task completion that contributors could help fulfill. Contributors, in this case, act as workers performing a manual task or labor that cannot be automated or automatically collected from the sensors on their smartphones. Tasks could include tagging buildings and routes in a city, e.g. CityExplorer [28], searching and identifying some pictures, e.g. CrowdSearch [29].

\subsubsection{The Who Genes}

Regarding the question of $\boldsymbol{w h o}$ is contributing and/or benefiting from the contributions, we identified three major answers:

- crowd: In this case, there is no explicit requester. The boundary between the requester and contributors is somewhat blurred as anyone on the platform can contribute and/or benefit from the contributions. Examples include mobile and situated crowdsourcing systems that help people socialize (opinionizer [30], WhozThat [31]), traffic related mobile crowdsourcing systems in which the same people are contributors of data and beneficiaries of other people's contributions (e.g., Tiramisu [11], waze [32], etc.).

- requester/contributor: In this case, beneficiaries make explicit requests for contribution and contributors directly respond to these in exchange for money (e.g., MoneyBee [33], Txteagle [25], etc.) or other types of rewards (snacks in Umati [34]). Requester and the contributor are easily distinguished in these systems, and their roles involved in one contribution are not interchangeable. Contributors are mostly motivated by monetary incentives and other types of immediate rewards. 
- practitioner/crowd: In this case, requester is a practitioner or a researcher, such as policy makers, medical doctors, etc. Unlike the requester/contributor case, where the motivation for making contribution is mainly monetary gains or other material rewards, in this case, the main motivation for contribution is the desire by the crowd to help the practitioner make changes that affect the crowd as a whole (e.g., an improved living environment like in Ubinion [7], VoiceYourView [35]) or individual contributor (e.g., improved health management like in MPCS [36]).

\subsubsection{The How Genes}

For the question of how the contributions are generated, there are primarily two approaches: opportunistic and participatory. The main difference between the two is whether it is the users that proactively contribute to system, or the system that collects users' information initially.

- participatory: Contributions are generated from and made by the users of the system. Such kind of mobile or situated crowdsourcing systems are usually in the model of requester/contributor, where requester(s) publish some tasks and contributors provide their wisdom or effort to complete these tasks. Hence in our proposed genome, most of them are also service-centric. For instance, CrowdSearch [29] requires its users' information retrieval behavior as well as Amazon Mechanical Turkers' validation. It should also be noted that situated crowdsourcing systems are all participatory because they require users to actively contribute.

- opportunistic: Contributions are generated, computed and collected from various sensors on mobile devices without the users' active involvement. A particular issue with this is the various privacy risks involved [37]. For instance, one risk is the leakage of user identity. Such a risk has already been identified in Amazon Mechanical Turk [38], and we posit it is even more probable to happen in mobile crowdsourcing because smartphones usually store a lot of user's identifiable information. Both participatory and opportunistic data collection could result in data leakage. Another risk could be a result of tracking user location 
and motion. Smartphones are equipped with various sensors that can easily track users' location and even their behavioral motion data. For instance, NoiseTube [39] uses sensors on smartphones to detect noise spots but could potentially use them to profile a user's frequent commutes or locations. More risk can be due to lack of privacy awareness. Users might assume that they are anonymous in crowdsourcing yet in fact they can be de-anonymized [40]. When data is being collected opportunistically in mobile crowdsourcing, user's privacy awareness may be even lower, and might compromise their privacy information without notice or considering consequences. For example, EmotionSense [41] can actively collect an individual's emotion data, which can be an important tool to profile her personality.

\subsubsection{The Why Genes}

In terms of why people are contributing to mobile and situated crowdsourcing systems, we found two main types of incentives:

- intrinsic motivations: These are derived by the contributors from making contributions, without any explicit incentives from the beneficiaries of the contributions. Intrinsic motivations include altruism (e.g., in askus [42]), a sense of social responsibility (e.g., in Ubinion [7], VoiceYourView [35], etc.) or fun (e.g., in Game of Words [6], Urbanopoly [43], etc.). When there is no clear differentiation between contributors and beneficiaries, people will contribute because of the value they derive from the system when they are beneficiaries. Thus, reciprocity is an important motivation in these systems (e.g., in BikeNet [44], CityExplorer [28], etc.). Broadly speaking, social responsibility seems to be a good incentive for systems that have the practitioner/crowd gene, whereas systems with the crowd gene may benefit from reciprocity: people contribute because they also benefit from others' contributions to the systems.

- extrinsic motivations: When there is differentiation between contributors and beneficiaries, and contributors do not derive any direct or indirect benefits from contributing, it is important to give them explicit incentives, such as money (e.g., 
in CrowdSearch [29], MobileWorks [45], etc.) or other valuable items like food (e.g., in Umati [34]).

Though diverse incentives, such as entertainment and ethical, are identified in mobile crowdsourcing applications [1], they are also discussed as intrinsic incentives in online communities (Kraut Resnick, 2012) along with those genes (money, love, and glory) that constitute the why genome of collective intelligence.

\subsubsection{The Where Genes}

Regarding the question of where the contribution is valid, we can clearly distinguish two new genes unique to mobile and situated crowdsourcing systems:

- local: Here we find mobile crowdsourcing systems that place a value on the location from which the contributions are made, either because the contributions are about the locations (e.g., Laermometer [24], Walkie-Markie [46], etc.) or because it is necessary to be in certain places to be able to contribute (e.g., CrowdMAC [47], TRAC [48]). Thus, contributions with the local gene are geo-tagged, with the location information becoming part of the contributions themselves.

- anywhere: Other crowdsourcing systems do not place a value on the location from where the users make contributions because the contributions are not location based. Examples in this category include Social Drive [27], VizWiz [49], etc.

\subsubsection{The When Genes}

Regarding the question of when the contribution is valid, we identified two answers as well.

- time-bound: These systems usually require or favor users that make contributions within a certain period of time and/or the contribution has a limited lifespan. The time when the contribution is made could be an integral part of the contribution. For instance, ParkNet [26] is a typical example of such system, because the availability of a parking spot is limited to a certain period of time. 
- anytime: These are systems that do not have requirement or constraint on the time of the contributions. A typical example is Walkie-Markie [46] that uses crowdsourcing to detect indoor WiFi signals. As long as the WiFi network has been setup in a building, there is no particular constraint on how fast a user can find a signal but what is more important is to detect and map it.

\section{Survey of the Systems Using the Proposed Genes}

In this section, we present our survey of 40 mobile and situated crowdsourcing systems by organizing them using the proposed genes. We introduce the systems by pairing up the where (i.e., local and anywhere) and when (i.e., time-bound and anytime) genes, which results in four pairs. Within each pair, we group the systems according to their what genes.

\subsection{Local and Time-bound}

This pair includes most of the mobile systems that crowdsource information about locations. Each contribution is also characterized by its temporal nature. Examples of this type of information include mapping noise levels (Laermometer [24] and NoiseTube [39]), collecting traffic information (Tiramisu [11] and SignalGuru [50], Waze [32]), finding available parking spots (ParkNet [26]), etc.

Contributions to the systems above are about locations and thus have the environmentcentric gene. Other systems, however, help people to fulfill or complete requests for service submitted by users (Airbnb [51], couchsurfing [52], CrowdMAC [47], Lyft [53] and Uber [54]). Thus, the contributions to these systems have the service-centric gene. Two other applications (Opinionizer [30] and WhozThat [31]) collect information about people to ease the process of socializing; giving their contributions the people-centric gene. Below we case study some sample systems for each what gene.

\subsubsection{Environment-centric}

Laermometer is a mobile crowdsourcing system that utilizes the crowd to map noisy spots with users' comments. This map helps users circumvent any noisy areas or go there to check what is happening [24]. In Laermometer, the contributions 
from users have both the local and time-bound genes because noisy spots are located in specific areas and might only exist for a certain period of time.

Similar to Laermometer, NoiseTube also leverages sensors on smartphones to enable users target and annotate noisy locations collectively to build a noise map [39]. NoiseTube, however, emphasizes more on the collaborative creation of the noise map. Just like Laemometer, contributions to NoiseTube have both the local and time-bound genes in order to develop a most updated noise map.

A number of applications harness the crowd to collect traffic related information. For example, Tiramisu is designed to report real-time bus transit information such as schedules, delays, routes and more based on users collaborative information report and contribution [11]. Similar to Tiramisu, SignalGuru leverages users mobile phones to collaboratively report traffic conditions and predict traffic signals and schedules [50]. Because bus transit information changes quickly over space and time, contributions to both systems have the local and time-bound genes.

ParkNet equips vehicles with a GPS device and an ultrasonic rangefinder to opportunistically gather parking information. This information is uploaded to a server that builds a real-time map of parking availability [26]. Because information about parking availability is dependent on location and varies over time, ParkNet contributions have both the local and time-bound genes.

\subsubsection{Service-centric}

This gene comprises the bulk of crowdsourcing systems. For example, Askus is a mobile crowdsourcing system that lets users ask contributors questions about locations. The system then sends these questions to people who are at that location [42]. For example, a user can ask people at the mall whether a particular shop is offering discounts. Askus will then forward this question to someone currently at the mall who is able to provide an answer. Similar to Askus is TRAC, a mobile crowdsourcing platform through which requesters can submit sensing tasks at particular locations. As an example, a user is able to ask pollution levels for a particular location. TRAC will then send this task to mobile phones that are at that location [48]. Because Askus and TRAC place a particular emphasis on the location of a potential contributor as well as 
on his timeliness in providing answers, we can consider contributions made on these platforms to have both the local and time-bound genes.

CrowdMAC creates a marketplace for mobile Internet access by allowing users to sell part of their mobile data plan to a nearby user of the CrowdMAC in need of immediate Internet access [47]. To be able to contribute or sell part of his / her data plan, a user has to be at the same place and at the same time as another CrowdMAC user willing to buy this data plan. Thus, contributions to CrowdMAC have both the local and time-bound genes.

Uber [54] and Lyft [53] let people share rides. Using these applications, a user can request a ride and a nearby available driver is able to connect with the user and fulfill his request for a fee. For these systems to work, it is critical that a driver be available at the time and location specified by the user. Therefore, the contributions to these platforms have the local and time-bound genes.

Couchsurfing [52] and Airbnb [51] are two popular applications through which people are able to share (Couchsurfing) or rent (Airbnb) their apartments to other people. Guests make requests to available hosts through the platforms. Thus, the time and place of availability of housing is necessary to accept these requests; giving these contributions the local and time-bound genes.

\subsubsection{People-centric}

Opinionizer is a situated crowdsourcing system developed to ease meeting and interaction between participants at social gatherings. Opinionizer was used at a book launch party, where participants could post comments and opinions that were projected on a big screen for other participants to see. With more participants converging around the system, this created a "honey-pot effect" that eased socializing and interaction among participants [30]. As the system was deployed for a specific occasion (book launch) that took place within a defined period of time, contributions to Opinionizer can be said to have had both the local and time-bound genes. Also helping people introduce themselves to each other, WhozThat is an application that leverages wireless technologies (Bluetooth and WiFi) on mobile phones and online social media sites such as Facebook to introduce people to each other. Users who are close to each 
other can receive information that answers the common but essential question, "Who's that?" [31]. Because users have to be in the same location and at the same time for the system to work, we consider it to have both the local and time-bound genes.

\subsection{Local and Anytime}

A few mobile and situated crowdsourcing systems prefer contributions to be made locally or in certain areas or places, but don't impose strict constraints on when the contribution should be made. Such systems carry the local and anytime genes and are very distributed in regard to other genes. The contributions to these systems can be environmental-centric, people-centric, or service-centric. The characteristic of such systems can be either participatory or opportunistic. In terms of application genres, such systems usually belong to geographic mapping, e.g. city exploration; or indoor mapping, e.g. indoor WiFi signal surveying. Below we show some sample systems for each what gene.

\subsubsection{Environment-centric}

CityExplorer is based on a mobile game that crowdsources user knowledge on geospatial information for applications like a location-based service [28] . The contributions to the system are made in a specific city, or target certain areas of a city. However, users are free to provide contributions at any time because geo-spatial changes or development of a city takes longer time. Similar to CityExplorer, Urbanopoly collects users information about urban venues through gamification features. The users can verify, collect, or report data about the urban venues to the system or share it to their social networks [43]. Since urban venues are location based, and do not change immediately, we regard contributions to Urbanoply as having the local and anytime genes. mCroud adds some gamification features and utilizes various sensors on iPhones to accomplish geographical tasks, such as geographical location-aware image collection [55].

Pazl is designed for indoor WiFi signal monitoring. It leverages users mobile phone sensors to automatically detect WiFi signals in an indoor environment, such as inside a building. Users contribute to each other by scanning the nearest or strongest WiFi in an indoor environment [56]. Because the WiFi spots are at specific areas inside a building 
and do not quickly change over time, contributions to Pazl have both the local and anytime genes. Walkie-Markie is also implemented for indoor use. It crowdsources WiFi spots sensing on users mobile phones. Besides that, it also gathers users' trajectory data to generate maps of pathways for an indoor environment so that users with no prior knowledge of the inner structure of a building can find their way [46].

Game of Words is a situated crowdsourcing system that is used to build dictionaries

of keywords describing different locations [6]. To encourage contributions, Game of Words is designed as a game that shows words to contributors and lets them decide whether the word describes the current location. Because Game of Words lets people play / contribute at any time, contributions to this system have the local and anytime genes.

\subsubsection{Service-centric}

Bazaar is a situated crowdsourcing platform that allows researchers to deploy situated crowdsourcing tasks [5]. Unlike the other situated crowdsourcing systems cited above, Bazaar is not a single purpose system but a marketplace that can host crowdsourcing tasks. Depending on the nature of the tasks, these can be completed at any time giving them the local and anytime genes.

Umati is a situated crowdsourcing system that rewards people with snacks in exchange for grading students' exams. Umati is a touchscreen system attached to a vending machine, which was originally placed in the Computer Science (CS) building of the University of California, Berkeley, to tap into the advanced knowledge of CS professors and students to grade CS related exams. Because it has a specific location but no rigid requirement on the time of contribution, we regard it to have the local and anytime genes, as well as the service-centric.

\subsection{Anywhere and Anytime}

The vast majority of mobile crowdsourcing systems that let users contribute from anywhere (anywhere gene) and at any time (anytime gene) are those that put in touch people who need certain tasks completed by a large crowd and do not particularly care where and when these tasks are completed. Thus, in addition to the anywhere and 
anytime genes, these systems also have the requester/contributor and participatory genes.

\subsubsection{Service-centric}

Service-centric platforms provide contributors with tasks that need to be completed, whether transcribing or translating a text, ranking photos, etc. Thus, we remark that their contributions have the service-centric gene.

For example, Txteagle enables people in developing countries to earn small amounts of money by completing tasks submitted by companies [25]. Some of these tasks include text translation, transcription, and surveys on the system. These tasks are from various corporations that pay the users back in either airtime or MPESA (mobile money) [25]. MoneyBee leverages mobile operators to reach a vast number of users, who are also the subscribers of the mobile operators. MoneyBee crowdsources its users' skills and preferences to complete the various small tasks in the system [33]. Like txteagle, mClerk sends tasks to mobile phone users via SMS to make it accessible to low-end mobile phone owners. mClerk was tested in India and was used to digitize documents in local languages [57]. On the other hand, instead of using text messages, MobileWorks relies on web browsers of low-end mobile phones to enable people perform optical character recognition (OCR) related tasks. These include transcribing chunks of documents that are sent as images [45]. These four applications (txteagle, MoneyBee, mClerk and MobileWorks) aim to help people in developing countries (or emerging markets) make money completing crowdsourcing tasks using their mobile phones. The contributions to these platforms have both the anywhere and anytime genes since the tasks provided do not have to be completed at specific locations and/or time.

Twitch is an application that enables its users to make micro-contributions when they unlock their phones. These micro-contributions include authoring a census of local human activity, rating stock photos and extracting structured data from Wikipedia pages [18]. Since users complete these tasks whenever they unlock their phones, which can be anywhere and at any time, contributions to Twitch have both the anywhere and anytime genes. 
Rankr aims at collecting and comparing opinions on ideas or photos. For example, the system is being used to compare and poll on the photos taken by users and even some commercial ideas, e.g. a poll that helps a company to identify the best way to improve employee satisfaction [58]. Because these tasks can be performed at any time and from any place, the contributions can be said to have both the anywhere and anytime genes.

Another example is CrowdSearch [29]. It asks its users to do information retrieval for search task and then uses Amazon Mechanical Turk to recruit another crowd of workers to validate the information retrieval results. Since the search tasks can be done from anywhere and at anytime, contributions CrowdSearch have the anywhere and anytime genes.

\subsubsection{People-centric}

These systems collect information that is related to people and their contributions are said to have the people-centric gene.

For example, Social Drive gathers users' driving behavioral data and recommends them the optimal driving habits for fuel economy on specific trips. The recommendation is provided based on the standard vehicular On-Board Diagnosis (OBD) and the rating and feedback from the crowd of users [27]. The driving behavioral data is usually independent of the location from which the contribution is made. Also, the behavioral data is collected over a long period of time instead of overnight, giving these contributions the anywhere and anytime genes. Also, since Social Drive collects data automatically without active user's participation, it has the opportunistic gene.

Mobile-phone based Patient Compliance System (MPCS) is mobile phone system that monitors and helps patients stick to their treatment regimen. It has a module that detects non-compliance by patients and can report this to their physicians, family members and peer community [59]. Since this monitoring process is always ongoing as long as the patient is undergoing treatment, independent of where he is, we consider contributions to MPCS to have the anytime and anywhere genes. 


\subsection{Anywhere and Time-bound}

Very few mobile or situated crowdsourcing systems that emphasize contributions to be time-bound but lets these contributions to be made anywhere. One exception is VizWiz [49], which requires answering questions in real time for people with visual impairment. Using VizWiz, a visually impaired person can take a photo of an object and ask people what the object is. People on VizWiz are able to answer from anywhere; however, the timeliness of these answers is critical. Another example is EmotionSense [41]. EmotionSense uses sensors on mobile phones to detect people's emotions for research in psychology. The microphone is used to collect voice samples, the accelerometer to detect user's activity, Bluetooth to scan mobile phones of nearby people, the GPS transceiver to locate the user, etc. Using these data points, EmotionSense is able to compute the user's emotions, send the data to social psychology researchers and, in the long-term, aims to provide users with real-time feedback and psychological help. Since people's emotions change throughout the day, contributions to EmotionSense have a limited lifespan. Thus, contributions to both VizWiz and EmotionSense have the anywhere and time-bound genes.

We believe there is an opportunity for mobile and situated crowdsourcing systems that have the anywhere and anytime genes to increase contributions by using the timebound gene with the anytime gene. For instance, the system could have rewards whose value diminishes over time.

\section{Discussion}

In this section, we first discuss the observed patterns of different genes using our survey results, which help reveal the design spaces that are underexploited. We then introduce new design suggestions that may increase user contributions to mobile and situated crowdsourcing systems. Our reflections show that the proposed genome model can be used to generate 1) ideas for new systems by combining the different genes, and 2) new design suggestions based on the interactions between the different genes. 


\subsection{Patterns Observed and Design Opportunities}

For each gene, we looked at all the systems that have that particular gene to discover if there were any common patterns of it combinations with other genes. Below, we report the major findings of our observations.

\subsubsection{Who}

When it comes to the who question, the requester/contributor gene has the most number of systems among those we reviewed (21 systems in total), followed by the crowd gene (14 systems) and the practitioner/crowd (5 systems).

All the requester/contributor systems we reviewed usually have the extrinsic genes (involving monetary incentives) and have the participatory gene. None of them uses the opportunistic gene. This is understandable as most crowdsourcing tasks with the requester/contributor gene require the active participation of the contributors to accomplish the task, whether to translate a text, identify an image, etc. However, this pattern suggests to explore if there is space to design systems that have the requester/contributor gene and the opportunistic gene. An example of such application could be a mobile agent system that distributes computational tasks to mobile phones [60]. These would be tasks that do not require interventions from the mobile phone users. A similar computational model has been studied in the field of sensor networks [61] but not much in mobile crowdsourcing.

The crowd gene has a large variety of combinations with other genes. More specifically, we have 9 systems that have the environment-centric gene and 5 systems with the people-centric gene; 8 systems have the time-bound gene, while 5 systems have the anytime gene; and 8 systems have the opportunistic gene and 7 systems have the participatory gene.

However, in our list of 40 reviewed systems, there was only one system with both the crowd and anywhere genes, i.e. Social Drive [27]. It seems that researchers did not give enough attention to designing this type of systems. People may explore other application systems having these two genes for collecting and sharing different types of information. For example, one can imagine a crowdsourcing system that helps people who want to lose or gain weight, or people who need emotional support, encourage 
each other. Besides allowing people to send each other supportive messages, users could also enter their daily calorie intake and be shown anonymous calorie intake of other members with the same body characteristics (height, weight, etc.) and health goals. Users who need emotional support could also share their strategies on how to regulate their emotions. This would encourage them to self-control more effectively. A system like this would have the crowd gene, since there are no explicit requesters, but everyone is a beneficiary of everyone's contributions. It would also have the anywhere gene, since people can contribute from wherever they are. These ideas can be built upon existing social systems that do not yet have crowdsourcing feature, e.g., Emotion Map, a mobile app for people to log their emotions [62].

There are very few systems with the practitioner/crowd gene (only 5 in our list of reviewed systems), even though an increasing number of agencies are trying to use mobile applications to engage the crowd to advance general knowledge. This suggests that we should design more applications for practitioners to help them in decision mak-

ing for all kinds of issues. For example, libraries are facing a service transition as they try to create new librarianship by developing community-driven library services [63]. Their current mobile applications are mainly for patron users to search book collections. It would be interesting to apply mobile and situated crowdsourcing to advance their new service design.

\subsubsection{How}

Among the genes that constitute the how question, the participatory gene has almost four times the number of systems ( 29 systems) as the opportunistic gene ( 7 systems). Besides these systems that have a single how gene, either participatory or opportunistic, very few systems combine both genes: MPCS [36], Social Drive [27], Tiramisu [11] and Waze [32].

This suggests that the participatory gene could be used to complement systems with the opportunistic gene, and vice-versa. The participatory gene could be used to collect information that cannot be obtained opportunistically, or it could be used to correct data collected opportunistically. For example, Tiramisu uses smartphone sensors to gather information on the movement of public transportation buses to predict 
their schedule. However, since it can only collect this information for users who are on the bus, Tiramisu has a participatory gene, allowing users who are not riding a bus to report ones they have spotted. Thus, this allows an optimum collection of data through both genes. On the other hand, one can also imagine opportunistic systems using the participatory gene to let users verify and correct data. For example, an application that collects data about the environment (temperature, pollution, noise, etc.) using a smartphone sensors could have a feature whereby they allow users to correct the data collected. It is a well-known fact that sensors are error prone. Thus, letting users correct the data collected could potentially increase its accuracy.

Because opportunistic systems use sensors to collect data without users' active involvement, when combined with the anywhere gene, these systems may pose more privacy concerns, especially when they also have the people-centric gene. This could explain why we have a relatively small number of such systems. In our list of 40 reviewed systems, only EmotionSense [41], MPCS [36] and Social Drive [27] fall under this category (with EmotionSense [41] being the only system that is purely opportunistic).

\subsubsection{What}

Close to half of the systems we reviewed have the service-centric gene (19 systems), followed by environment-centric (12) and people-centric (9) genes.

All the service-centric systems have the requester/contributor gene. The contribution to most of the service-centric systems require some computation from contributors. Very few systems are labor based (e.g., Uber). However, these systems are poised to become more popular with the rise of the sharing economy [64]. As these systems become popular, there will be more safety and service quality assurance issues. For instance, for people from disadvantaged communities, such as those unemployed, certain sharing economy applications could elicit safety and privacy concerns [65].

All environment-centric systems are also local. Designers of environment-centric systems may consider leveraging both participatory and opportunistic approaches to increase the service coverage of contributions. For example, in Tiramisu, the design of spotting and tracing a bus leverages users movements around the bus stop areas and 
along the bus lines [22]. An opportunistic tracing is about a contributors own trajectory or experience, while a participatory spot is about what can be observed nearby. Such approaches can potentially work with other sensing systems. For example, in Waze [32], spotting a police officer is similar to spotting a bus in Tiramisu. In fact, reporting where a user received a ticket could be a good method for tracing a users bus ride. Both could indicate location information of police officers in that area. Similarly, those systems that collect noise level information usually rely on users to carry the sensors to the noisy location. Adding a participatory sensing approach, a new user interaction could be added for users to spot party events at their neighborhood area, which may help estimate the noise level too.

\subsubsection{Where}

For the where question, the local gene has as much as twice the number of systems (27 systems) as the anywhere gene (13 systems). The local gene also has more combinations with other genes in our surveyed systems than the anywhere gene. For example, there are 13 systems with the crowd gene, 11 systems with the requester/contributor gene and 3 systems with the practitioner/crowd gene. The local gene combines with all three what genes, e.g., there are 12 local systems with the environment-centric gene, 7 have the people-centric gene, while 9 have the service-centric gene.

Though we have 13 anywhere systems in our list of surveyed systems, only 1 system has the opportunistic gene (EmotionSense [41]), 3 have the time-bound gene (VizWiz [49], Crowd Translator [66] and EmotionSense [41]) and 3 have the peoplecentric gene (EmotionSense [41], MPCS [36] and Social Drive [27]).

\subsubsection{When}

Among the genes of the when question, the anytime gene has more combination with other genes. With the exceptions of EmotionSense [41], VizWiz [49] and Crowd Translator [66], all the systems surveyed that have the time-bound gene also have the local gene as well. If well designed, the time-bound gene can help increase contributions to systems with a varied number of genes through the use of deadlines. For example, a system (e.g., rankr [58]) with the anytime gene could consider using the time-bound 
gene to temporarily boost the number of contributions through, for example, the use of time-bound competitions and time-bound rewards.

\subsection{Design Suggestions for Encouraging User Contributions}

We reviewed the 35 design claims for encouraging user contributions to online systems one by one, and discussed how the new genes of mobile and situated crowdsourcing systems can inspire new design suggestions from the existing design claims. We now present how the genome model indicate design implications in two directions. One direction is to leverage the opportunity provided by unique genes (e.g., the local, requester/contributor or opportunistic genes) to expand or adapt the proposed design claims of online communities to mobile and situated crowdsourcing. The other direction is to apply variations of design claims to tackle the challenges of mobile and situated crowdsourcing raised by the unique genes, e.g., the opportunistic, anytime, and practitioner/crowd genes.

\subsubsection{Who}

For systems that have either the practitioner/crowd or the crowd/researcher gene, designers may consider providing performance feedback to increase contribution. A study by Locke et al. showed that participants' knowledge of how they were doing on a task had a significant impact on their performance [67]. Locke divided his subjects into two groups and gave them a complex computation task to perform. After the first stage of the task was completed, one group was given its score on the first stage and the other group was not given its score. The two groups were then asked to set goals for the second phase of the experiment. The results were that the group that received feedback set more difficult goals than the second group; and the first group's performance on the second phase of the experiment was significantly higher than that of the second group. Elaborating further on this research, Erez showed that knowledge of how one was faring on a task is a "necessary condition for goals to affect performance". Kraut's design claim 15 for increasing contribution to online crowdsourcing systems also states that, "goals have larger effects when people receive frequent feedback about their performance with respect to the goals" [10]. 
However, many of the practitioner/crowd crowdsourcing systems do not have this mechanism yet. Potentially, one reason is that it usually takes time for practitioners or researchers to aggregate the collected contributions, do analysis, and making policy changes or decisions. The importance and effectiveness of providing feedback to their contributors can be easily overlooked.

Similar ideas have been studied in public relations and communications [68, 69]. For example, studies on the interactions between the practitioners, e.g. public service provider, and the public shows that different strategies impact user's interactions significantly on social media [70]. In order to receive user contributions, practitioners should provide means two way communication. This will be particularly important when crowdsourcing crime information from the public [71]. The public was often found discouraged for not receiving feedback on how the crime was investigated or if the criminals were caught after sharing crime related information with the police officers. Thus, system designers should develop different strategies to provide better transparency of the practitioners and the researchers. The effectiveness of this design suggestion in terms of encouraging user contributions effectively needs to be further evaluated in mobile and situated crowdsourcing.

\subsubsection{How}

For systems that have the opportunistic gene, designers can consider providing users a context-aware tool for tracking contributions that are needed. The design claim 2 states that, "easy to use tools for finding and tracking work that needs to be done will increase the amount that gets done" [10]. A number of online communities provide these tools to their users, such as Wikipedia's "watchlist" [72], Bugzilla [73] used by many open source development projects to send alerts and provide advance search features for their community members to keep track of changes, etc. When a system has the opportunistic gene, contributors may not be aware of their contributions or easily lose track of it. Using a user's location context (home, work, entertainment place, etc.), his mobility context (driving, walking, using a bus, stationary, etc.), powerful tracking and notifications tools may be developed for mobile crowdsourcing systems to improve the awareness of users' opportunistic contributions. An analogy could be showing users 
their caloric burn on the Fitbit application could improve people's awareness with their shared activities and encourage them to have more effective diet control.

For systems that have the participatory gene, system designers may also consider sending requests for contributions to perspective contributors based on their location. This suggestion is inspired by the design claim 4 in [10], i.e. "compared to broadcasting requirements for contribution to all community members, asking specific people to make contributions increases the likelihood that they will". This design claim becomes especially important for mobile systems if notifications are used to convey these requests. There have been various notification systems designed for this purpose [74]. Also, research has shown that users constantly receive a lot of notifications on their mobile phones and a large number of notifications is not appreciated by users and can have an inverse effect [75]. In particular, Shirazi et al. found that the "frequency of notifications when the information given is not perceived as important, is a crucial factor that leads to increased blacklisting" [75]. Thus, one way to reduce the number of requests is by targeting specific contributors based on criteria such as location context (e.g., work, home, mall, etc.), activity context (e.g., walking, not moving, running, etc.). This may further materialize the privacy preserving mechanisms proposed in iSafe (Ballesteros, J. et al. 2012), an algorithm to evaluate the safety of a user based on her location data. Our design suggestion could be used to emphasize the importance and protection of the contributions, e.g. incidence reports, from vulnerable users in certain dangerous districts.

\subsubsection{Where}

For the where question, the local gene is specific to mobile and situated crowdsourcing systems; thus, we present design suggestions for this gene. For example, one design suggestion could be to show users how many other nearby contributors have responded to a request. Numerous studies have shown that people are influenced by the decisions made by others when making their own decisions. This has long been exploited by marketers to influence the purchasing decisions of buyers through ratings, list of bestsellers, customers' recommendations, etc. Hoffer noted that "when people are free to do as they please, they usually imitate each other" [76]. Banerjee called 
this "herd behavior", when everyone is doing what everyone else is doing [77]. To increase people's contributions to online crowdsourcing systems, in the design claim 12, Kraut also notes that "people are more likely to comply with a request when they see that other people have also complied" [10]. To exploit the "herd behavior" in mobile crowdsourcing systems, we suggest showing contributors the number of people who have responded to a request for contribution. In location sensitive mobile crowdsourcing systems specifically, people might be more compelled to contribute when they see that other people in their vicinity have contributed. This design suggestion could be embedded into some gamification features for mobile crowdsourcing system design to increase the timeliness, quantity, and quality of responses.

Another design suggestion for systems that have the local gene could be to design interfaces that show the contributor the location of the beneficiaries of his/her contribution. People were found more likely to contribute when the system interface showed which groups of users were going to benefit from these contributions [78]. Users of MovieLens, a movie recommendation system, were $3.7 \%$ more likely to rate a movie when they were shown the value of their rating to other users of the system. The likelihood of contributing increased to $7.4 \%$ when the user identified with the beneficiary group or liked the said group (in this case the benefeciary group was made of users who enjoyed the same genre of movies as the contributor). Coupling this finding with Kraut's design claim 6 [10], for mobile crowdsourcing, we could therefore suggest designing interfaces that show the benefits of the user's contributions based on location context (workplace, home neighborhood, etc.), current vicinity or other shared interests.

\subsubsection{When}

For systems that have the anytime gene, designers may consider using deadlines sparingly to increase contributions while maintaining enjoyability of contributing. Kraut's design claim 14 encourages the use of deadlines to increase contributions, especially as the deadlines approaches [10]. It is surprising that among the 40 systems, only 2 of them have the anywhere and time-bound gene pair (Vizwiz [49] and EmotionSense [41]), whereas there are 14 systems that have the anywhere and anytime gene pair. 
However, if we apply deadlines to Rankr [58] or CrowdSearch [29], they can easily be transformed to have the time-bound gene, which may potentially increase user contributions as a result of the design claim of using deadline.

Although the immediate energizing effects of deadlines have been documented [79], an experiment conducted by Amabile et al. also showed that "externally imposed deadlines for completion of a task can have a decrement in subsequent intrinsic interest in that task" [80]. Their participants showed less interest in repeating a task (in this instance, playing a word game) when they had previously done it under an externally imposed deadline. Considering this possibility, we may divide potential contributors into different groups. According to the particular type of requests for contributions (either because of their location context, activity context, prior contributions, etc.), requests could be sent to the groups alternatively. In addition, different rewards may be given not only based on the quantity of contributions but also based on how quickly the contributors respond. Such design suggestion may work better for the systems that have the service-centric gene, such as: Askus [42], CrowdSearch [29], etc..

\subsection{Limitations and Future Work}

The proposed genome was created by studying 40 systems. This list of 40 systems is by no means exhaustive, but to our best effort, represents a good sample of the current status. We expect new genes to emerge as more diverse mobile or situated systems are built. In fact, we believe the model will work more effectively if new genes are created or new design principles are added to address the unique characteristics of the genes or gene pairs.

We reviewed the 35 design claims for encouraging user contributions to online systems one by one, and discussed how the new genes of mobile and situated crowdsourcing systems can inspire new design suggestions from the existing design claims. The design suggestions presented above are derived from our own literature review and thought process and would need to be further validated by running user studies. In our future work, we will test and validate the effectiveness of different design suggestions that can promote user contributions in different gene settings. 


\section{Conclusions}

In this paper, we surveyed 40 mobile and situated crowdsourcing systems. We defined six fundamental questions regarding user contributions by drawing inspiration from the MIT's genetic model of collective intelligence. We identified 14 new genes for mobile and situated crowdsourcing systems and presented the 40 systems using our genome model. The novel approach of analyzing mobile and situated crowdsourcing systems allows us to make many interesting observations of the patterns of the genes, and reflect on how the unique genes promote new design opportunities. We also discuss new design suggestions driven by the genes and existing design principles for encouraging user contributions to online communities. By applying the proposed genome model, system designers could identify where their respective mobile and situated crowdsourcing systems fit in and therefore inform their design decisions. Systems designers can also spot categories of mobile and situated crowdsourcing systems that are under-exploited by existing applications.

\section{Acknowledgements}

This material is based upon work supported by the National Science Foundation under Grant No.1464312.Any opinions, findings, and conclusions or recommendations expressed in this material are those of the author(s) and do not necessarily reflect the views of the National Science Foundation. We also thank the support from a Google Faculty Research Award.

[1] G. Chatzimilioudis, A. Konstantinidis, C. Laoudias, D. Zeinalipour-Yazti, Crowdsourcing with smartphones, Internet Computing, IEEE 16 (5) (2012) 36-44. doi:10.1109/MIC.2012.70.

[2] J. Goldman, K. Shilton, J. Burke, D. Estrin, M. Hansen, N. Ramanathan, S. Reddy, V. Samanta, M. Srivastava, R. West, Participatory sensing: A citizenpowered approach to illuminating the patterns that shape our world, Foresight $\&$ Governance Project, White Paper (2009) 1-15. 
[3] B. Guo, Z. Wang, Z. Yu, Y. Wang, N. Y. Yen, R. Huang, X. Zhou, Mobile crowd sensing and computing: The review of an emerging human-powered sensing paradigm, ACM Computing Surveys (CSUR) 48 (1) (2015) 7.

[4] D. Geiger, M. Rosemann, E. Fielt, Crowdsourcing information systems: a systems theory perspective, in: Proceedings of the 22nd Australasian Conference on Information Systems (ACIS 2011), 2011.

[5] S. Hosio, J. Goncalves, V. Lehdonvirta, D. Ferreira, V. Kostakos, Situated crowdsourcing using a market model, in: Proceedings of the 27th annual ACM symposium on User interface software and technology, ACM, 2014, pp. 55-64.

[6] J. Goncalves, S. Hosio, D. Ferreira, V. Kostakos, Game of words: Tagging places through crowdsourcing on public displays, in: Proceedings of the 2014 conference on Designing interactive systems, ACM, 2014, pp. 705-714.

[7] S. Hosio, V. Kostakos, H. Kukka, M. Jurmu, J. Riekki, T. Ojala, From school food to skate parks in a few clicks: using public displays to bootstrap civic engagement of the young, in: Pervasive Computing, Springer, 2012, pp. 425-442.

[8] J. Goncalves, D. Ferreira, S. Hosio, Y. Liu, J. Rogstadius, H. Kukka, V. Kostakos, Crowdsourcing on the spot: altruistic use of public displays, feasibility, performance, and behaviours, in: Proceedings of the 2013 ACM international joint conference on Pervasive and ubiquitous computing, ACM, 2013, pp. 753-762.

[9] T. W. Malone, R. Laubacher, C. Dellarocas, The collective intelligence genome, IEEE Engineering Management Review 38 (3) (2010) 38.

[10] R. E. Kraut, P. Resnick, S. Kiesler, Y. Ren, Y. Chen, M. Burke, N. Kittur, J. Riedl, J. Konstan, Building Successful Online Communities: Evidence-Based Social Design, The MIT Press, 2012.

[11] J. Zimmerman, A. Tomasic, C. Garrod, D. Yoo, C. Hiruncharoenvate, R. Aziz, N. R. Thiruvengadam, Y. Huang, A. Steinfeld, Field trial of tiramisu: crowdsourcing bus arrival times to spur co-design, in: Proceedings of the SIGCHI Conference on Human Factors in Computing Systems, ACM, 2011, pp. 1677-1686. 
[12] N. D. Lane, E. Miluzzo, H. Lu, D. Peebles, T. Choudhury, A. T. Campbell, A survey of mobile phone sensing, Comm. Mag. 48 (9) (2010) 140-150. doi : $10.1109 / \mathrm{MCOM} .2010 .5560598$. URL http://dx.doi.org/10.1109/MCOM.2010.5560598

[13] S. Tilak, Real-world deployments of participatory sensing applications: Current trends and future directions, ISRN Sensor Networks 2013.

[14] U. Lee, M. Gerla, A survey of urban vehicular sensing platforms, Computer Networks 54 (4) (2010) 527-544.

[15] F. Fuchs-Kittowski, D. Faust, Architecture of mobile crowdsourcing systems, in: Collaboration and Technology, Springer, 2014, pp. 121-136.

[16] M. Vukovic, R. Das, S. Kumara, From sensing to controlling: the state of the art in ubiquitous crowdsourcing, International Journal of Communication Networks and Distributed Systems 11 (1) (2013) 11-25.

[17] A. Tomasic, J. Zimmerman, A. Steinfeld, Y. Huang, Motivating contribution in a participatory sensing system via quid-pro-quo, in: Proceedings of the 17th ACM Conference on Computer Supported Cooperative Work \&\#38; Social Computing, CSCW'14, ACM, New York, NY, USA, 2014, pp. 979-988. doi:10.1145/ 2531602.2531705 .

URL http://doi.acm.org/10.1145/2531602.2531705

[18] R. Vaish, K. Wyngarden, J. Chen, B. Cheung, M. S. Bernstein, Twitch crowdsourcing: crowd contributions in short bursts of time, in: Proceedings of the $32 \mathrm{nd}$ annual ACM conference on Human factors in computing systems, ACM, 2014, pp. 3645-3654.

[19] T. Yan, B. Hoh, D. Ganesan, K. Tracton, T. Iwuchukwu, J.-S. Lee, Crowdpark: A crowdsourcing-based parking reservation system for mobile phones, University of Massachusetts at Amherst Tech. Report.

[20] B. Frei, Paid crowdsourcing, Current State \& Progress toward Mainstream Business Use, Smartsheet. com Report, Smartsheet. com 9. 
[21] A. Tomasic, J. Zimmerman, A. Steinfeld, Y. Huang, Motivating contribution in a participatory sensing system via quid-pro-quo, in: Proceedings of the 17th ACM conference on Computer supported cooperative work \& social computing, ACM, 2014, pp. 979-988.

[22] Y. Huang, A. Tomasic, J. Zimmerman, A. Steinfeld, Combining contribution interactions to increase coverage in mobile participatory sensing systems, Mobile$\mathrm{HCI}$, under review.

[23] T. Erickson, Geocentric crowdsourcing and smarter cities: Enabling urban intelligence in cities and regions, A position paper for the 1st International Workshop on ubiquitous crowdsourcing.

[24] M. Bilandzic, M. Banholzer, D. Peev, V. Georgiev, F. Balagtas-Fernandez, A. De Luca, Laermometer: a mobile noise mapping application, in: Proceedings of the 5th Nordic conference on Human-computer interaction: building bridges, ACM, 2008, pp. 415-418.

[25] N. Eagle, txteagle: Mobile crowdsourcing, in: Internationalization, Design and Global Development, Springer, 2009, pp. 447-456.

[26] S. Mathur, T. Jin, N. Kasturirangan, J. Chandrasekaran, W. Xue, M. Gruteser, W. Trappe, Parknet: drive-by sensing of road-side parking statistics, in: Proceedings of the 8th international conference on Mobile systems, applications, and services, ACM, 2010, pp. 123-136.

[27] X. Hu, V. Leung, K. G. Li, E. Kong, H. Zhang, N. S. Surendrakumar, P. TalebiFard, Social drive: a crowdsourcing-based vehicular social networking system for green transportation, in: Proceedings of the third ACM international symposium on Design and analysis of intelligent vehicular networks and applications, ACM, 2013, pp. 85-92.

[28] S. Matyas, C. Matyas, C. Schlieder, P. Kiefer, H. Mitarai, M. Kamata, Designing location-based mobile games with a purpose: collecting geospatial data with 
cityexplorer, in: Proceedings of the 2008 international conference on advances in computer entertainment technology, ACM, 2008, pp. 244-247.

[29] T. Yan, V. Kumar, D. Ganesan, Crowdsearch: exploiting crowds for accurate real-time image search on mobile phones, in: Proceedings of the 8th international conference on Mobile systems, applications, and services, ACM, 2010, pp. 7790.

[30] Y. Rogers, H. Brignull, Subtle ice-breaking: encouraging socializing and interaction around a large public display, in: Workshop on Public, Community. and Situated Displays, Citeseer, 2002.

[31] A. Beach, M. Gartrell, S. Akkala, J. Elston, J. Kelley, K. Nishimoto, B. Ray, S. Razgulin, K. Sundaresan, B. Surendar, et al., Whozthat? evolving an ecosystem for context-aware mobile social networks, Network, IEEE 22 (4) (2008) 50-55.

[32] waze, Free community-based mapping, traffic navigation app, [Online; accessed 23-September-2015] (2015).

URL https://www.waze.com/

[33] D. Govindaraj, N. KVM, A. Nandi, G. Narlikar, V. Poosala, Moneybee: Towards enabling a ubiquitous, efficient, and easy-to-use mobile crowdsourcing service in the emerging market, Bell Labs Technical Journal 15 (4) (2011) 79-92.

[34] K. Heimerl, B. Gawalt, K. Chen, T. Parikh, B. Hartmann, Communitysourcing: engaging local crowds to perform expert work via physical kiosks, in: Proceedings of the SIGCHI Conference on Human Factors in Computing Systems, ACM, 2012, pp. 1539-1548.

[35] J. Whittle, W. Simm, M.-A. Ferrario, K. Frankova, L. Garton, A. Woodcock, J. Binner, A. Ariyatum, et al., Voiceyourview: collecting real-time feedback on the design of public spaces, in: Proceedings of the 12th ACM international conference on Ubiquitous computing, ACM, 2010, pp. 41-50.

[36] G. C. G. Chen, B. Y. B. Yan, M. S. M. Shin, D. Kotz, E. Berke, MPCS: Mobilephone based patient compliance system for chronic illness care, 2009 6th Annual 
International Mobile and Ubiquitous Systems: Networking \& Services, MobiQuitousdoi:10.4108/ICST.MOBIQUITOUS2009.6829.

[37] Y. Wang, Y. Huang, C. Louis, Respecting user privacy in mobile crowdsourcing, ASEScience 2 (2) (2013) pp-50.

[38] M. Lease, J. Hullman, J. P. Bigham, M. S. Bernstein, J. Kim, W. Lasecki, S. Bakhshi, T. Mitra, R. C. Miller, Mechanical turk is not anonymous, Available at SSRN 2228728.

[39] N. Maisonneuve, M. Stevens, M. E. Niessen, L. Steels, Noisetube: Measuring and mapping noise pollution with mobile phones, in: Information Technologies in Environmental Engineering, Springer, 2009, pp. 215-228.

[40] T. Kandappu, V. Sivaraman, A. Friedman, R. Boreli, Exposing and mitigating privacy loss in crowdsourced survey platforms, in: Proceedings of the 2013 workshop on Student workhop, ACM, 2013, pp. 13-16.

[41] K. K. Rachuri, M. Musolesi, C. Mascolo, P. J. Rentfrow, C. Longworth, A. Aucinas, Emotionsense: a mobile phones based adaptive platform for experimental social psychology research, in: Proceedings of the 12th ACM international conference on Ubiquitous computing, ACM, 2010, pp. 281-290.

[42] S. Konomi, N. Thepvilojanapong, Askus: Amplifying mobile actions, Pervasive Computing 5538 (2009) 202-219. doi: 10 . 1007/978-3-642-01516-8.

[43] I. Celino, D. Cerizza, S. Contessa, M. Corubolo, D. Dell'Aglio, E. D. Valle, S. Fumeo, Urbanopoly-a social and location-based game with a purpose to crowdsource your urban data, in: Privacy, Security, Risk and Trust (PASSAT), 2012 International Conference on and 2012 International Confernece on Social Computing (SocialCom), IEEE, 2012, pp. 910-913.

[44] S. B. Eisenman, E. Miluzzo, N. D. Lane, R. A. Peterson, G.-S. Ahn, A. T. Campbell, Bikenet: A mobile sensing system for cyclist experience mapping, ACM Transactions on Sensor Networks (TOSN) 6 (1) (2009) 6. 
[45] P. Narula, P. Gutheim, D. Rolnitzky, A. Kulkarni, B. Hartmann, Mobileworks: A mobile crowdsourcing platform for workers at the bottom of the pyramid., Human Computation 11 (2011) 11.

[46] G. Shen, Z. Chen, P. Zhang, T. Moscibroda, Y. Zhang, Walkie-markie: Indoor pathway mapping made easy, in: Proceedings of the 10th USENIX conference on Networked Systems Design and Implementation, USENIX Association, 2013, pp. 85-98.

[47] N. Do, C.-H. Hsu, N. Venkatasubramanian, Crowdmac: a crowdsourcing system for mobile access, in: Proceedings of the 13th International Middleware Conference, Springer-Verlag New York, Inc., 2012, pp. 1-20.

[48] Z. Feng, Y. Zhu, Q. Zhang, L. M. Ni, A. V. Vasilakos, Trac: Truthful auction for location-aware collaborative sensing in mobile crowdsourcing, in: INFOCOM, 2014 Proceedings IEEE, IEEE, 2014, pp. 1231-1239.

[49] J. P. Bigham, C. Jayant, H. Ji, G. Little, A. Miller, R. C. Miller, R. Miller, A. Tatarowicz, B. White, S. White, T. Yeh, Vizwiz: Nearly real-time answers to visual questions, in: Proceedings of the 23Nd Annual ACM Symposium on User Interface Software and Technology, UIST' 10, ACM, New York, NY, USA, 2010, pp. 333-342. doi:10.1145/1866029.1866080. URL http://doi.acm.org/10.1145/1866029.1866080

[50] E. Koukoumidis, L.-S. Peh, M. R. Martonosi, Signalguru: leveraging mobile phones for collaborative traffic signal schedule advisory, in: Proceedings of the 9th international conference on Mobile systems, applications, and services, ACM, 2011, pp. 127-140.

[51] Airbnb, Vacation rentals, homes, apartments rooms for rent - airbnb, [Online; accessed 23-September-2015] (2015).

URL https: / / www.airbnb.com/

[52] couchsurfing, Stay with locals and make travel friends - couchsurfing, [Online; 
accessed 23-September-2015] (2015).

URL https://www. couchsurfing.com/

[53] lyft, A ride whenever you need one - lyft, [Online; accessed 23-September-2015] (2015).

URL https://www. Iyft.com/

[54] uber, Uber — sign up to drive or tap and ride, [Online; accessed 23-September2015] (2015).

URL https://www. uber.com/

[55] T. Yan, M. Marzilli, R. Holmes, D. Ganesan, M. Corner, mcrowd: a platform for mobile crowdsourcing, in: Proceedings of the 7th ACM Conference on Embedded Networked Sensor Systems, ACM, 2009, pp. 347-348.

[56] V. Radu, L. Kriara, M. K. Marina, Pazl: A mobile crowdsensing based indoor wifi monitoring system, in: Network and Service Management (CNSM), 2013 9th International Conference on, IEEE, 2013, pp. 75-83.

[57] A. Gupta, W. Thies, E. Cutrell, R. Balakrishnan, mClerk: enabling mobile crowdsourcing in developing regions, Proc. CHI '12 (2012) 1843-1852doi: $10.1145 / 2207676.2208320$.

URL http://doi.acm.org/10.1145/2207676.2208320

[58] Y. Luon, C. Aperjis, B. A. Huberman, Rankr: A mobile system for crowdsourcing opinions, in: Mobile computing, applications, and services, Springer, 2011, pp. $20-31$.

[59] G. Chen, B. Yan, M. Shin, D. Kotz, E. Berke, Mpcs: Mobile-phone based patient compliance system for chronic illness care, in: Mobile and Ubiquitous Systems: Networking Services, MobiQuitous, 2009. MobiQuitous '09. 6th Annual International, 2009, pp. 1-7. doi:10.4108/ICST.MOBIQUITOUS2009.6829.

[60] D. B. Lange, Mobile objects and mobile agents: The future of distributed computing?, Springer Berlin Heidelberg, Berlin, Heidelberg, 1998, pp. 1-12. doi : 
$10.1007 / \mathrm{BEb} 0054084$.

URL http://dx.doi.org/10.1007/BFb0054084

[61] C. L. Fok, G. C. Roman, C. Lu, Mobile agent middleware for sensor networks: an application case study, in: IPSN 2005. Fourth International Symposium on Information Processing in Sensor Networks, 2005., 2005, pp. 382-387. doi: $10.1109 /$ IPSN.2005.1440953.

[62] Y. Huang, Y. Tang, Y. Wang, Emotion map: A location-based mobile social system for improving emotion awareness and regulation, in: Proceedings of the 18th ACM Conference on Computer Supported Cooperative Work \&\#38; Social Computing, CSCW '15, ACM, New York, NY, USA, 2015, pp. 130-142. doi:10.1145/2675133.2675173.

URL http://doi.acm.org/10.1145/2675133.2675173

[63] A. S. K.-S. C. Jackson, Y. Huang, Face-to-face matters: Inspirations from the human library, in: Special Issue of the International Journal of Mobile HCI, 2015.

[64] J. Hamari, M. Sjöklint, A. Ukkonen, The sharing economy: Why people participate in collaborative consumption, Journal of the Association for Information Science and Technology.

[65] T. R. Dillahunt, A. R. Malone, The promise of the sharing economy among disadvantaged communities, in: Proceedings of the 33rd Annual ACM Conference on Human Factors in Computing Systems, ACM, 2015, pp. 2285-2294.

[66] J. Ledlie, B. Odero, E. Minkov, I. Kiss, J. Polifroni, Crowd translator: on building localized speech recognizers through micropayments, ACM SIGOPS Operating Systems Review 43 (4) (2010) 84-89.

[67] E. A. Locke, J. F. Bryan, Goal-setting as a determinant of the effect of knowledge of score on performance, The American Journal of Psychology (1968) 398-406.

[68] I. Mergel, A framework for interpreting social media interactions in the public sector, Government Information Quarterly 30 (4) (2013) 327-334. 
[69] A. Meijer, M. Thaens, Social media strategies: Understanding the differences between north american police departments, Government Information Quarterly 30 (4) (2013) 343-350.

[70] Y. Huang, S. Huo, Y. Yao, N. Chao, Y. Wang, J. Grygiel, S. Sawyer, Municipal police departments on facebook: What are they posting and are people engaging?, the 17th Annual International Conference on Digital Governmental Research.

[71] E. Tan, H. Xia, C. Ji, R. V. Joshi, Y. Huang, Designing a mobile crowdsourcing system for campus safety, iConference 2015 Proceedings.

[72] Wikipedia, Help:watchlist - wikipedia, the free encyclopedia, [Online; accessed 23-September-2015] (2015).

URL https://en.wikipedia.org/wiki/Help:Watchlist

[73] Bugzilla, Bugzilla, [Online; accessed 23-September-2015] (2015).

URL https: //www.bugzilla.org/

[74] D. S. McCrickard, M. Czerwinski, L. Bartram, Introduction: Design and evaluation of notification user interfaces, Int. J. Hum.-Comput. Stud. 58 (5) (2003) 509-514. doi:10.1016/S1071-5819(03)00025-9. URL http://dx.doi.org/10.1016/S1071-5819(03)00025-9

[75] A. Sahami Shirazi, N. Henze, T. Dingler, M. Pielot, D. Weber, A. Schmidt, Largescale assessment of mobile notifications, in: Proceedings of the SIGCHI Conference on Human Factors in Computing Systems, ACM, 2014, pp. 3055-3064.

[76] E. Hoffer, The passionate state of mind, Buccaneer Books, 1996.

[77] A. V. Banerjee, A simple model of herd behavior, The Quarterly Journal of Economics (1992) 797-817.

[78] A. M. Rashid, K. Ling, R. D. Tassone, P. Resnick, R. Kraut, J. Riedl, Motivating participation by displaying the value of contribution, in: Proceedings of the SIGCHI conference on Human Factors in computing systems, ACM, 2006, pp. 955-958. 
[79] L. A. Arvedson, Deadlines and organizational behavior: A laboratory investigation of the effect of deadlines on individual task performance., Ph.D. thesis, ProQuest Information \& Learning (1975).

[80] T. M. Amabile, W. DeJong, M. R. Lepper, Effects of externally imposed deadlines on subsequent intrinsic motivation., Journal of personality and social psychology 34 (1) (1976) 92. 